Stefanie Thate*

\title{
Kinder über das Vorlesen für naturwissenschaftlich-technische Themen begeistern
}

https://doi.org/10.1515/bfp-2020-0015

Zusammenfassung: Viele naturwissenschaftliche und technische Phänomene lassen sich über spannende Geschichten erklären und erzählen. Wissenschaftliche Studien zeigen, dass sich gerade im Grundschulalter durch die Beschäftigung mit mathematisch-naturwissenschaftlichtechnischen Fragestellungen die Sprach- und Lesekompetenz fördern lässt und umgekehrt.

MINT-Vorlesepaten begeistern Kinder zwischen drei und zehn Jahren über das Vorlesen geeigneter Lesematerialien (Bücher und Apps) und Anschlussaktionen für MINT-Themen und fördern ihre Sprach- und Lesekompetenz.

Schlüsselwörter: MINT-Vorlesepaten; Sprach- und Leseförderung; Experimente

\section{Spark Children's Interest in STEM Subjects by Reading Aloud}

Abstract: Most children's books are full of STEM themes: plants, animals, numbers, and more. Natural and technological phenomena can be easily explained in reading aloud sessions and experienced through simple experiments. By reading aloud to them on a regular basis volunteer readers support children aged three to ten in learning about science and technology and simultaneously improve language and reading skills.

Keywords: STEM Volunteer readers; language and reading skills improvement; experiments

Was passiert bei einem Vulkanausbruch und warum schwimmt ein großes Schiff, aber ein kleiner Stein geht unter? Viele naturwissenschaftliche und technische Phänomene lassen sich über spannende Geschichten erklären und erzählen.

Studien zeigen, dass sich gerade im Grundschulalter durch die Beschäftigung mit mathematisch-naturwissen- schaftlich-technischen Fragestellungen die Sprach- und Lesekompetenz fördern lässt und umgekehrt. Das Forschen führt zu einem großen Interesse an sprachlichen und kommunikativen Mitteln, denn die Kinder wollen untersuchte Phänomene benennen, beschreiben, Vermutungen ausdrücken.

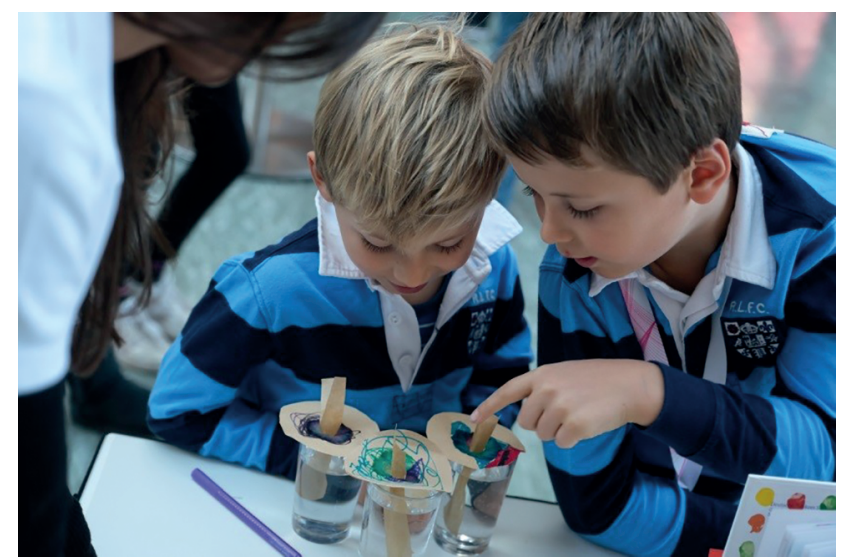

Abb. 1: MINT-Vorleseaktion zum Thema Farben

\section{Das Projekt MINT-Vorlesepaten}

Hier setzt die Kooperation der Deutsche Telekom Stiftung mit der Stiftung Lesen in Deutschland an. Ehrenamtler engagieren sich seit Dezember 2015 regelmäßig in Kindergärten, Grundschulen und Bibliotheken - einige als alleinige Vorleser, andere als Vorleser-Teams, um die zeitliche Belastung für den Einzelnen zu begrenzen. In manchen Bibliotheken bieten neben Ehrenamtlern auch hauptberufliche Bibliothekare MINT-Vorleseaktionen für Kinder ${ }^{1}$ im Alter von drei bis zehn Jahren an.

Für die Anschlussaktionen nach dem Vorlesen werden Alltagsmaterialien verwendet, die in einem durchschnittlichen Haushalt vorhanden sind. So lassen sich zum Beispiel mit Mehl und Bällen Mondkrater nachstellen

1 www.telekom-stiftung.de/mint-vorlesepaten. 
oder mit Essig und Backpulver Vulkane imitieren. Die einfache Vorbereitung und die Freude, die MINT-Vorleseaktionen dem Vorleser und den Kindern bereiten, haben sie zu einem festen Bestandteil des Angebots in zahlreichen Bibliotheken, Kindergärten und Grundschulen werden lassen.

MINT-Vorlesepaten schaffen ein niedrigschwelliges Angebot, um Kinder über das Vorlesen für MINT-Themen $\mathrm{zu}$ begeistern. Diese Kombination von geeigneten Lesematerialien (auch digitale Angebote) und spannenden, interaktiven Anschlussaktionen lässt sich in jeder Muttersprache umsetzen. Deshalb hat die Telekom Stiftung gemeinsam mit lokalen Partnern Kooperationen in Albanien, Polen, der Republik Moldau und in Rumänien geschlossen. Die genaue Ausgestaltung variiert von Land $\mathrm{zu}$ Land: so sind in Deutschland vorrangig Ehrenamtler als Vorleser tätig, während in der Republik Moldau, in Rumänien und Polen Bibliothekare die MINT-Vorleseaktionen durchführen.

Insgesamt engagieren sich inzwischen rund 2900 MINT-Vorlesepaten in verschiedenen Ländern.

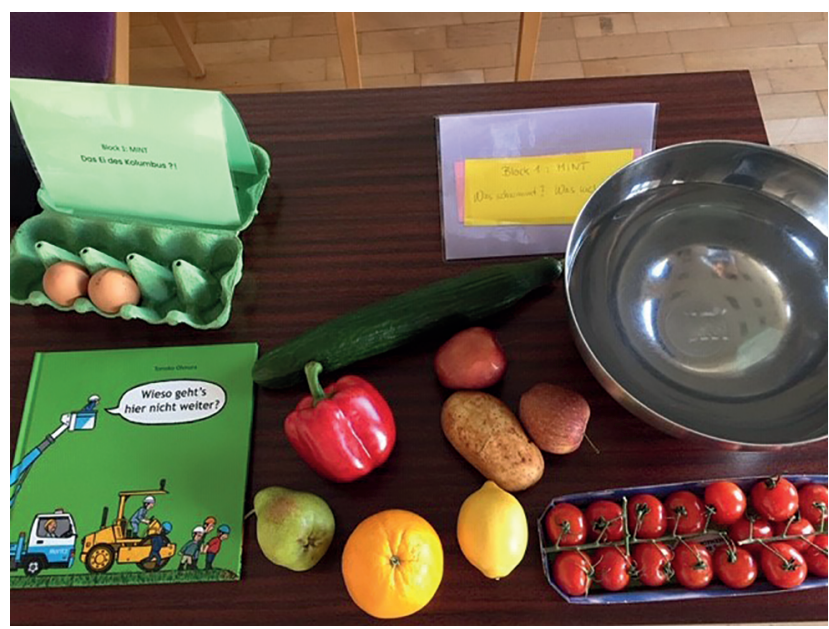

Abb. 2: Das Küchenlabor umfasst Alltagsmaterialien

\section{MINT-Vorlesepaten International}

Im November 2018 hat die Zusammenarbeit zwischen der Deutsche Telekom Stiftung und der rumänischen Progress Foundation begonnen. In der ersten Projektphase haben die beiden Stiftungen in 12 von insgesamt 41 Regionen ein niedrigschwelliges Angebot geschaffen, um Kinder in Rumänien über das Vorlesen für MINT-Themen zu begeistern. In der zweiten Projektphase konnten sich auch interessierte Bibliotheken aus den übrigen Regionen Rumäniens beteiligen.
Die konkrete Umsetzung von „Ora sa STIM“² (rumänischer Projektname) vor Ort lag bei der Progress Foundation. Trainer der rumänischen Stiftung haben in der ersten Projektphase rund 130 Bibliothekare darin geschult, wie sich das Vorlesen altersgerechter Kinderbücher mit MINT-Bezug um spannende Anschlussaktionen ergänzen lässt.

Seit Februar 2019 haben die Bibliothekare wöchentlich MINT-Vorleseaktionen für Kinder zwischen drei und zehn Jahren. Hierfür haben sie von der Progress Foundation entwickelte Materialien zu Medien- und Aktionstipps und kostenlose Bücherboxen erhalten. Großen Wert legte die Progress Foundation darauf, die Teilnehmer untereinander zu vernetzen und den Wissens- und Erfahrungsaustausch zu fördern. Die 234 Bibliotheken haben kontinuierlich über ihre Erfahrungen, Erkenntnisse und Herausforderungen bei der Durchführung der MINT-Vorleseaktionen in einer projekteigenen, aber öffentlich einsehbaren Facebook-Gruppe berichtet. Der Austausch erfolgte sehr engagiert und anschaulich. Neue Medien- und eigene Aktionstipps wurden in der Gruppe vorgestellt und zahlreiche Fotos und Videos ermöglichen eine einfache Nachahmung. Das Kooperationsprojekt endete im Mai 2020.

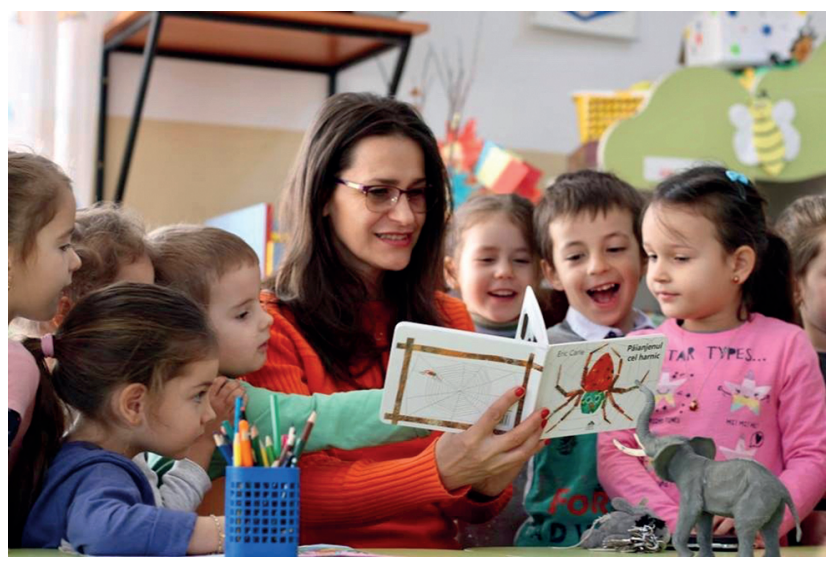

Abb. 3: MINT-Vorleseaktion in Rumänien

In Polen kooperiert die Telekom Stiftung seit Februar 2019 mit der polnischen Information Society Development Foundation (IFRS), um Kinder durch Vorlesen für mathematisch-naturwissenschaftliche Inhalte zu begeistern und ihre Sprach- und Lesekompetenz zu fördern.

Unter dem Titel „Para - buch! Książka w ruch!“ sind 100 Tandems aus Bibliothekaren und Ehrenamtlern in fünf

2 www.facebook.com/orasastim. 
von insgesamt 16 Regionen Polens ${ }^{3}$ gebildet und geschult worden. Seit dem Sommer 2019 bieten sie im Zweiwochenrhythmus MINT-Vorleseaktionen für Kinder zwischen drei und zehn Jahren in „MINT-Vorlese-Clubs“ in Bibliotheken an. Unterstützt werden sie durch Medien- und Aktionstipps sowie von Materialien, die von der IFRS Stiftung adaptiert und entwickelt wurden. Die Stiftung wird dabei von dem Museum of Children's Book, eine Abteilung der Regional Public Library Warschau und dem Center of Children's Literature, unterstützt. Als Freiwillige werden vor allem Menschen mit einem naturwissenschaftlich-technischen Hintergrund gewonnen, zum Beispiel Ingenieure oder Handwerker, die ihr Wissen und ihre Erfahrungen über MINT-Vorleseaktionen an Kinder weitergeben. Dieser starke Bezug zur Praxis führt zu großer Begeisterung bei den Kindern. Sie lernen direkt von den Profis und sind mit großem Eifer bei der Sache. Das Kooperationsprojekt läuft bis Ende 2020 und wird durch das Goethe-Institut ${ }^{4}$ Warschau mit Buchpaketen unterstützt.

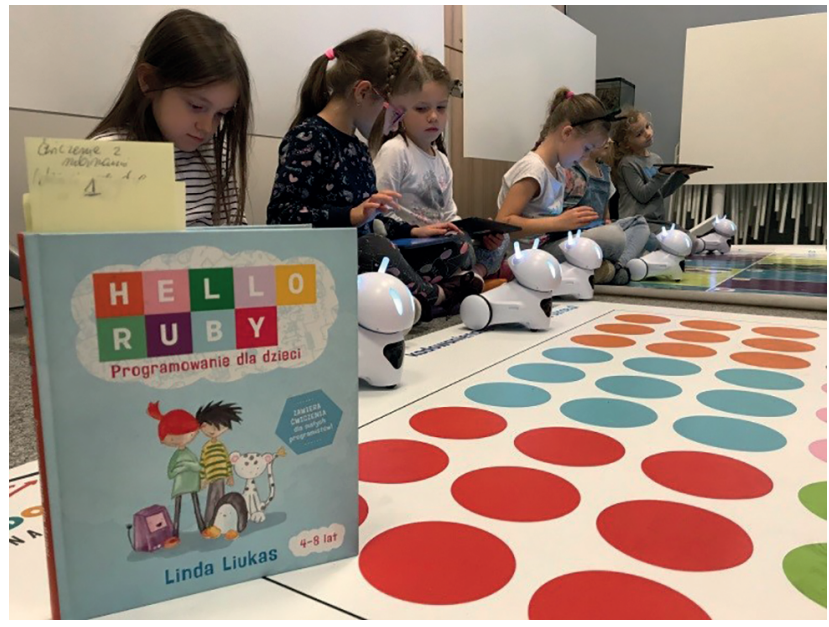

Abb. 4: MINT-Vorleseaktion in Polen

Mit den SOS-Kinderdörfern Weltweit arbeitet die Deutsche Telekom Stiftung in Albanien zusammen. Im Oktober 2019 haben die Vorbereitungen in den beiden SOS-Kinderdörfern in Tirana und Shkoder begonnen. Das Training der Mitarbeiter der SOS-Kinderdörfer und der Ehrenamtler fand am 23. und 24. April in Tirana statt. Die sukzessive Adaptierung vorhandener und die Identifikation neuer Materialien läuft parallel. Auch in Albanien wird der Austausch mit lokalen Akteuren gesucht. Erste Gespräche mit

3 http://frsi.org.pl/para-buch-ksiazka-w-ruch-pilotaz-projektu-prom ujacego-czytelnictwo/.

4 https://www.goethe.de/ins/sk/de/index.html. dem Goethe-Zentrum Tirana und der Zentralbibliothek haben bereits stattgefunden.

Über diese konkreten Kooperationen in Albanien, Polen, Republik Moldau und Rumänien hinaus, unterstützt die Deutsche Telekom Stiftung weitere Aktivitäten zur Verbindung von MINT und Sprache in Belgien, Österreich, ${ }^{5}$ der Slowakischen Republik, Spanien und Südtirol. Die Ansätze in den jeweiligen Ländern sind unterschiedlich.

In Österreich adressiert beispielsweise das Österreichische Bibliothekswerk über „MINT: lesen - sprechen tun“ Bibliothekare, Vorlesepaten, Eltern und Pädagogen, die kindliche Neugier durch das Vorlesen ausgewählter Medien und leicht zu realisierender Versuche zu wecken. Ergänzend wird Kindergärten und Grundschulen in Salzburg und Wien die Möglichkeit geboten, MINT-Themenboxen (Bücher plus Materialien plus Bilderbuchkinos) unentgeltlich auszuleihen.

Erste spannende Ansätze, naturwissenschaftliche Themen schon ganz früh in österreichischen Eltern-KindGruppen mitzudenken, hat das Forum Katholischer Erwachsenenbildung unter dem Titel „MINI-MINT“ entwickelt. Die Familien werden dabei als der Ort verstanden, in denen der Grundstein für die Bildungs-Begeisterung und im konkreten Fall für die MINT-Begeisterung - gelegt wird. In den meisten Gruppen werden bereits zahlreiche MINT-Themen altersgerecht und spielerischer berührt, z. B. mein Körper, Tiere, Farben. Darauf aufbauend ist die Handreichung „MINI-MINT: von Anfang an die Welt entdecken“ zu zehn verschiedenen Themen entwickelt worden.

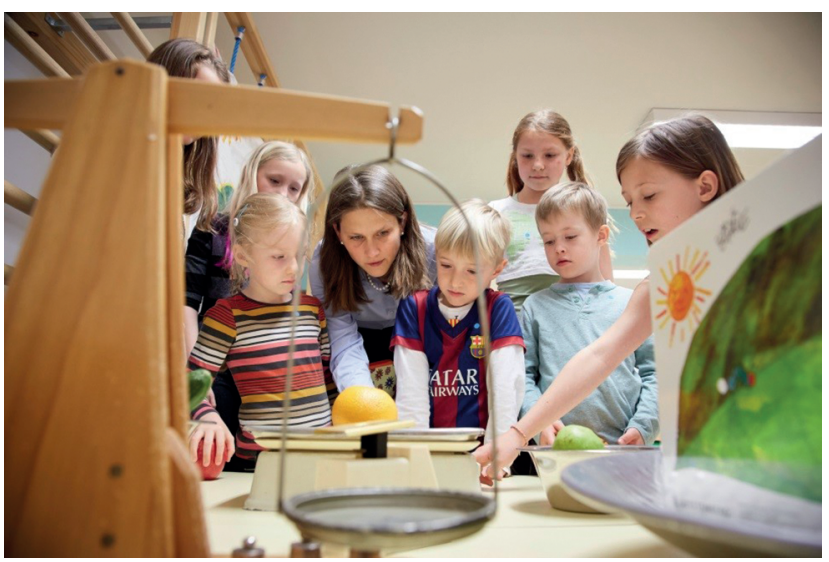

Abb. 5: MINT-Vorleseaktion in Österreich

Die gewinnbringende Verbindung von MINT und Sprache wurde in der Slowakischen Republik durch das Goethe-

5 http://www.biblio.at/blog/?p=2488. 


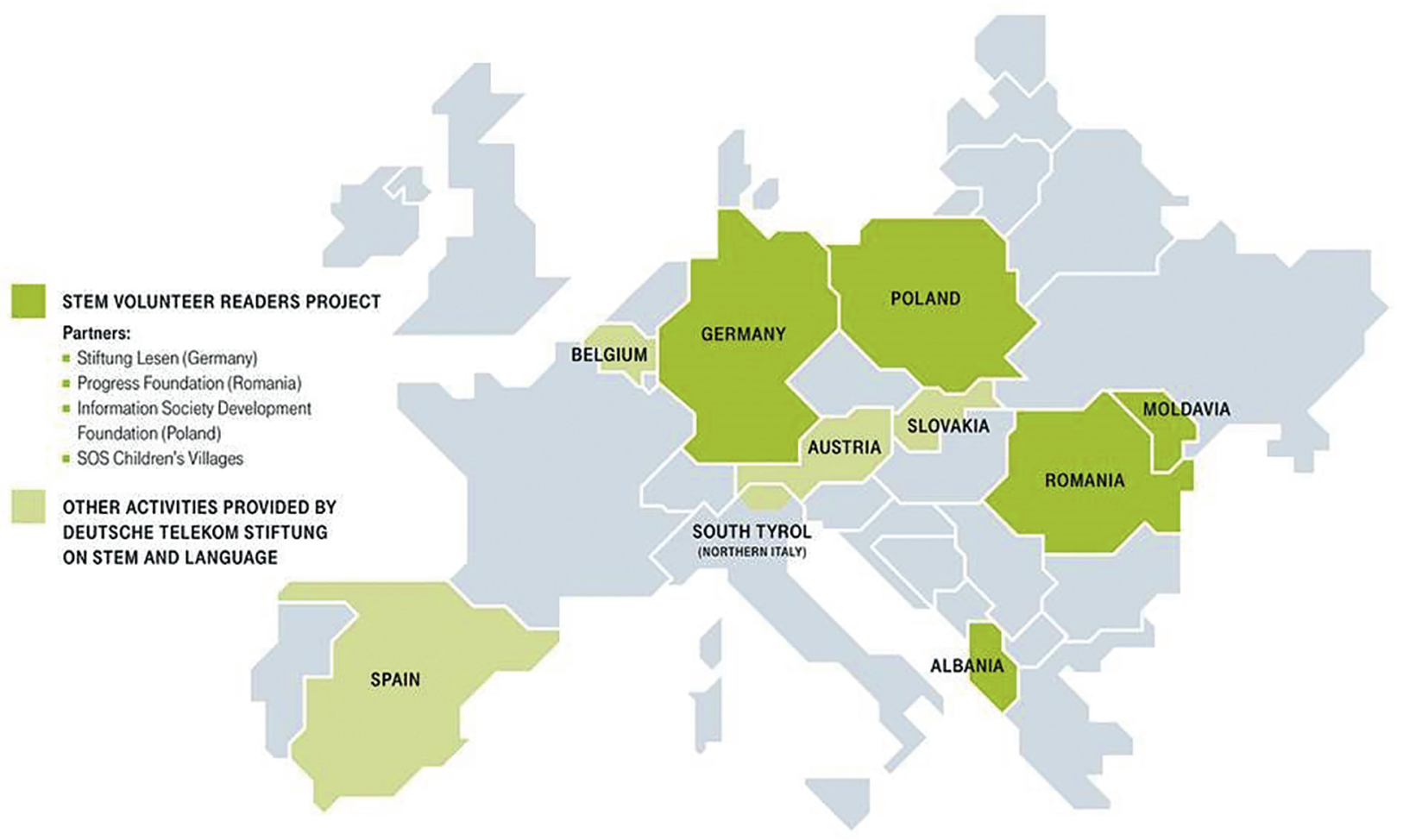

Abb. 6: Projektpartner der Projektpartner der Telekom-Stiftung

Institut Bratislava genutzt, um Kinder, die Deutsch als Fremdsprache lernen, zu unterstützen. Über anregende naturwissenschaftliche und technische Inhalte, die kreativ und spielerisch aufgegriffen werden, wird das Interesse an der „neuen“ Sprache gesteigert.

Die Beispiele verdeutlichen: Die Themengebiete MINT und Sprach- und Leseförderung lassen sich unabhängig von der Muttersprache niedrigschwellig an verschiedenen Orten und durch unterschiedliche Personen gewinn- und freudebringend miteinander verbinden. Gelingt es überdies, in Interaktion mit verschiedenen Akteuren (Vertreter von MINT-Berufen, Museen, Organisationen für Ehrenamt etc.) zu treten, können MINT-Vorleseaktionen zu einem lehrreichen, abwechslungsreichen und nachhaltigen Angebot werden.

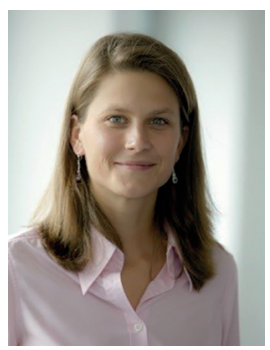

\section{Stefanie Thate}

Deutsche Telekom Stiftung

Projektleiterin MINT-Vorlesepaten

Friedrich-Ebert-Allee 71-77

D-53113 Bonn

stefanie.thate@telekom-stiftung.de 